\title{
Risk factors for lymph node metastasis of the left recurrent laryngeal nerve in patients with esophageal squamous cell carcinoma
}

\author{
Chuangui Chen, Zhao Ma, Xiaobin Shang, Xiaofeng Duan, Jie Yue, Hongjing Jiang^^ \\ Department of Minimally Invasive Esophagus Surgery, Key Laboratory of Prevention and Therapy, National Clinical Research Center of Cancer, \\ Tianjin Medical University Cancer Institute and Hospital, Tianjin, China \\ Contributions: (I) Conception and design: C Chen, H Jiang; (II) Administrative support: H Jiang; (III) Provision of study materials or patients: All \\ authors; (IV) Collection and assembly of data: Z Ma; (V) Data analysis and interpretation: All authors; (VI) Manuscript writing: All authors; (VII) \\ Final approval of manuscript: All authors. \\ Correspondence to: Hongjing Jiang, MD. Department of Minimally Invasive Esophagus Surgery, Key Laboratory of Prevention and Therapy, National \\ Clinical Research Center of Cancer, Tianjin Medical University Cancer Institute and Hospital, Tianjin 300060, China. Email: jianghjsci@163.com.
}

\begin{abstract}
Background: The factors for left recurrent laryngeal nerve (RLN) lymph node (LN) metastasis have important guiding significance for whether the left RLN LNs should be dissected in patients with esophageal squamous cell carcinoma (ESCC), but few studies are currently available. To analyze the risk factors of LN metastasis of the left RLN area and to assess which LNs should be dissected in ESCC.

Methods: This was a retrospective study of patients who underwent McKeown minimally invasive esophagectomy (MIE) (no neoadjuvant therapy) at Tianjin Medical University Cancer Institute and Hospital (from January 2016 to December 2019). The detection of left RLN LNs using enhanced computed tomography (CT) was compared with the pathological examination.

Results: Of the total 94 participants, 43 had LN metastasis. The metastatic LNs were mainly located next to left (18.1\%) and right (14.9\%) RLN, and the left gastric artery (13.8\%). Tumor size, LN size, tumor invasion ( $\mathrm{T}$ stage), $\mathrm{N}$ stage, and tumor node metastasis (TNM) stage were associated with left RLN LNs metastasis, while LN size was the only independently associated factor [odds ratio $(\mathrm{OR})=1.569,95 \%$ confidence interval (CI): 0.259-1.956, $\mathrm{P}=0.0012]$. The area under receiver operating characteristic (ROC) curve (AUC) reached 0.877 , with $64 \%$ sensitivity and $75 \%$ specificity using a cutoff of $5.5 \mathrm{~mm}$ LN size.

Conclusions: The size of left RLN LN is independently associated with metastasis. Left RLN LNs >5.5 $\mathrm{mm}$ at CT examination are more likely to be positive and should probably be dissected.
\end{abstract}

Keywords: Esophageal squamous cell carcinoma (ESCC); minimally invasive esophagectomy (MIE); lymph node (LN); recurrent laryngeal nerve (RLN); receiver operating characteristic

Submitted Dec 24, 2020. Accepted for publication Feb 08, 2021.

doi: $10.21037 / \mathrm{atm}-21-377$

View this article at: http://dx.doi.org/10.21037/atm-21-377

\section{Introduction}

Esophageal squamous cell carcinoma (ESCC) represents $90 \%$ of esophageal cancers in China and is a highly aggressive digestive tract tumor that is associated with a poor prognosis due to early lymph node $(\mathrm{LN})$ and distant metastases (1-4). The incidence of esophageal cancer was estimated at 572,034 cases in 2018 globally, with 508,585 deaths (5). Although esophagectomy has a curative effect, it carries significant morbidity and mortality risks. Not

$\wedge$ ORCID: 0000-0002-6053-283X. 
surprisingly, LN dissection plays an important role in the staging and radical treatment of esophageal cancer owing to the prognostic relevance of $\mathrm{LN}$ metastasis $(1,6)$. In esophageal cancer, LN metastasis, even at the early tumor invasion ( $\mathrm{T}$ stages), indicates poor prognosis (1-4). In addition, regardless of the depth of $\mathrm{T}$ stage, postoperative regional $\mathrm{LN}$ metastasis is associated with a dismal 5-year survival rate of $7 \%$ (7).

Therefore, safe and thorough LN dissection in esophageal cancer can reduce local tumor recurrence, prolong patient survival, and achieve accurate pathological staging (8-10). As to the lymphadenectomy of esophageal cancer, the concept of total mesenteric excision (TME) has been proposed, and the core of TME is dissection of the left recurrent laryngeal nerve (RLN) LNs (11). Mediastinal LNs, especially left RLN LNs, are the most frequent sites of metastasis in esophageal cancer $(12,13)$. Dissection of left RLN LNs by open esophagectomy has a high risk of injury that can lead to hoarseness, pneumonia, and even death (13). Although endoscopy-assisted surgery and Da Vinci robot-assisted minimally invasive esophagectomy (MIE) has significantly eased the dissection of left RLN $\mathrm{LNs}$, it is still associated with considerable risks and complications (14). Therefore, the identification of risk factors for left RLN LN metastasis has important guiding significance for whether the left RLN LNs should be dissected.

Preoperative contrast-enhanced computed tomography (CT) is routinely used to assess LN metastasis, which not only determines clinical staging in esophageal cancer $(15,16)$, but is also critical for optimal LN dissection $(16,17)$. However, there is currently no diagnostic standard to assess whether a given left RLN LN is metastatic or not on CT images. Many surgeons believe that superior mediastinal LNs $>1 \mathrm{~cm}$ in diameter must be dissected (18), this criterion is empirical, and other factors might need to be considered.

Therefore, the aim of this study was to explore the predictive factors of left RLN LN metastasis in patients with ESCC and use CT examination to assess which LNs must be dissected during Da Vinci robot-assisted and thoracic laparoscopic video-assisted McKeown MIE (19).

We present the following article in accordance with the STARD reporting checklist (available at http://dx.doi. org/10.21037/atm-21-377).

\section{Methods}

\section{Study design and participants}

This was a retrospective study of patients who underwent McKeown MIE at Tianjin Medical University Cancer Institute and Hospital from January 2016 to December 2019. All procedures performed in this study involving human participants were in accordance with the Declaration of Helsinki (as revised in 2013). The study was approved by the Ethics Committee of Tianjin Medical University Cancer Institute and Hospital (No. Bc2020176). The requirement for informed content was waived due to the retrospective nature of the study.

The inclusion criteria were as follows: (I) diagnosed with ESCC, (II) underwent MIE with the Da Vinci robotassisted and thoracic laparoscopic video-assisted approach, (III) underwent thoracic-abdominal 2-field LNs dissection via right thoracic approach with the neck as the esophagealgastric anastomosis site, and (IV) preoperative CT images available. Patients who underwent neoadjuvant therapy, open esophagectomy, or mediastinal LNs dissection without left RLNLNs were excluded (Figure 1). The demographic data were collected from medical records.

\section{CT examination of the left $R L N L N s$}

All participants underwent a CT scan (Brilliance 64 CT scanner; Philips Medical Systems, Best, Netherlands) of the chest and upper abdomen with intravenous contrast within 1 week before surgery. The scanning parameters were tube voltage of $120 \mathrm{kV}$, tube current of $80,150 \mathrm{~mA}$, collimation of $64 \times 0.625 \mathrm{~mm}$, pitch of 1.375 , slice thickness of $5 \mathrm{~mm}$, reconstruction slice thickness of $1 \mathrm{~mm}$, and slice spacing of $0.8 \mathrm{~mm}$. For enhanced scanning, $60-100 \mathrm{~mL}$ of non-ionic contrast agent (Onipex (Omnipaque, GE Healthcare Ireland Limited, Ireland) $300 \mathrm{mgI} / \mathrm{mL}$ ) was administered by the bolus injection method through the elbow vein, at a rate of $2.0-3.0 \mathrm{~mL} / \mathrm{s}$, and the scan was started following a $30 \mathrm{~s}$ pause after injection. The original data was reconstructed to a layer thickness of $1.25 \mathrm{~mm}$ and a layer interval of $1.00 \mathrm{~mm}$ to perform image processing and analysis. For the left RLN-LNs, the most typical (the largest and well-demarcated $\mathrm{LN}$ ) was selected and measured by 2 experienced radiologists ( $>10$ years of work experience). 


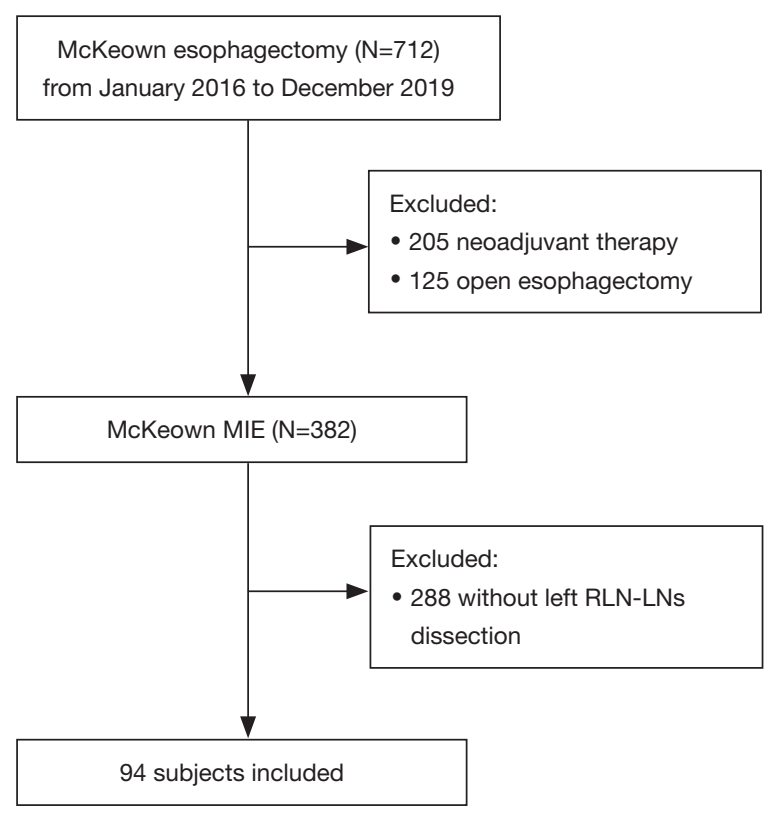

Figure 1 Patient flowchart. MIE, minimally invasive esophagectomy; RLN, recurrent laryngeal nerve.

\section{Pathological diagnosis}

The resected primary tumor and LN specimens were fixed and stained with hematoxylin and eosin and examined by 2 pathologists with $>10$ years of work experience. The participants with at least $1 \mathrm{LN}$ pathologically diagnosed as squamous cell carcinoma metastasis among the resected left RLNLNs were grouped as the LN-positive group. The others were grouped as the LN-negative group. The lymph node metastasis rate (LNMR) was defined as the proportion of participants with positive LN metastasis. The lymph node positive rate (LNPR) was defined as the ratio of the number of metastasis-positive LNs to the total number of LNs removed.

\section{Surgical approach of MIE}

Da Vinci robot-assisted (Da Vinci Si/Xi, Intuitive Surgical, Inc., Sunnyvale, CA, USA) and thoracic laparoscopic video-assisted MIE were performed in 3 stages. First, thoracoscopy or the Da Vinci robot was used to mobilize the esophagus and dissect the thoracic LNs in the prone position with the right hand holding the head. Second, thoracoscopy or the Da Vinci robot was used to create the gastric conduit and dissect the abdominal LNs in the supine position. Finally, a left cervical neck incision was made to create a cervical esophagogastric anastomosis. All surgeries were conducted by senior physicians with $>10$ years of work experience.

\section{The esophagus-mobilized retraction method to dissect left RLNLNS}

The left RLN runs through a cylinder-like region including soft tissues and LNs surrounded by the esophagus, main trachea, left common carotid artery, left subclavian artery, and the thoracic duct (Figure S1A) (20). The esophagusmobilized retraction method was used to dissect the left RLN LNs (13). The participants were anesthetized with a single lumen cannula to allow slight rotation of the trachea in order to visualize the left paratracheal groove. After mobilizing the esophagus, it was retracted to the right with a loop through the trocar port and fixed at the third intercostal space in the midaxillary line (Figure S1B). The trachea was rotated slightly to the right with a 5-lobed liver retractor to fully expose the left RLN region (Figure S1B). The soft tissues at the corner of the main trachea and the left main bronchus were first dissociated by electric hook, followed by the soft tissues along the main trachea up to the root of the neck at the lower thyroid or the lower thyroid artery. The pretracheal soft tissue was lifted in order to clearly expose the left RLN (Figure S1C). The beginning of the left RLN was visualized surrounding the aortic arch, and the soft tissues were hollowed out using forceps (Figure S1D). Finally, the soft tissues and LNs were dissected along the left RLN with an ultrasonic scalpel, scissors, or Maryland bipolar forceps to skeletonize the left RLN (Figure S1E,F).

\section{Statistical analysis}

The data are presented as frequency (\%) and were analyzed using the chi-square test. Multivariable logistic regression analysis was performed including tumor length, LN diameter, T stage, $\mathrm{N}$ stage, and TNM stage to identify independent predictive factors for left RLN LN metastases. The predictive power was measured using the area under the receiver operating characteristic (ROC) curve (AUC). All statistical analyses were performed using the software SPSS version 15.0 (SPSS Inc., Chicago, IL, USA). P values $<0.05$ were considered statistically significant. 


\section{Results}

\section{Participant characteristics}

Based on the eligibility criteria, 94 participants (81 males and 13 females, with a median age of 60 years) were included, of which $6,12,18,17,11,24$, and 6 were respectively pathological stages Ia, Ib, IIa, IIb, IIIa, IIIb, and IVa, according to the eighth edition of American Jount Committee on Cancer (AJCC) esophageal cancer staging $(21,22)$. There were 9 cases of upper thoracic esophageal cancer, 50 of middle thoracic esophageal cancer, and 35 of lower thoracic esophageal cancer (Table 1).

\section{Postoperative LNs yield and complications}

The LNMR among the thoracic ESCC patients was 45.7\%, with 43 out of the 94 participants showing LN metastasis. A total of 2,505 LNs were removed, of which 129 were positive, indicating a $5.3 \%$ LNPR. Among them, 17 had LN metastasis of left RLN (LNMR 18.1\%) (Table S1). In addition, 14 participants had LN metastasis of the right RLN (LNMR 14.9\%), cardiac LNs were positive in 11 participants (LNMR 11.7\%), and 13 had left gastric artery LN metastasis (LNMR 13.8\%). Therefore, the LNMR of left RLN was the highest among all LNs (Table S1). In addition, 3 upper para-esophageal, 4 middle paraesophageal, 7 lower para-esophageal, 2 left main bronchus, and 1 splenic artery LN metastases were recorded among the 43 participants. No metastases were observed in the subcarinal, celiac artery, and hepatic artery LNs. Taken together, the metastatic LNs of esophageal cancer were mainly located next to the left and right RLN, peri-cardiac tissues, and left gastric artery (Table S1).

There were 6 cases with vocal cord paralysis (16.38\%), 3 with anastomotic leakage (3.19\%), and 10 with pulmonary infection $(10.64 \%)$ after esophagectomy. No chylothorax and massive bleeding was reported. No deaths were recorded within 30 days of the operation.

\section{Risk factors for left RLN LN metastasis}

The clinicopathological factors affecting the LNMR at the left RLN are summarized in Table 1. Tumor size $(\mathrm{P}=0.0323)$, $\mathrm{T}$ stage $(\mathrm{P}=0.038), \mathrm{N}$ stage $(\mathrm{P}<0.001)$, TNM stage $(\mathrm{P}<0.001)$, and $\mathrm{LN}$ size $(\mathrm{P}<0.001)$ were associated with positive left RLN LN in the univariable analyses, but not age, gender, tumor location, or tumor differentiation (all $\mathrm{P}>0.05$ ). The multivariable logistic regression analysis
(Table 2) revealed that $\mathrm{LN}$ diameter was an independent risk factor for positive left RLN LNs (OR $=1.569,95 \%$ CI: $0.259-1.956, \mathrm{P}=0.0012$ ).

\section{Diagnostic value of CT for left RLN LN metastasis}

We used ROC curve analysis to evaluate the accuracy of preoperative CT in diagnosing left RLN LN metastasis based on the LN size. The AUC was 0.877 , which indicated an optimal diagnostic efficacy (Figure 2). In addition, the optimal cutoff value of $\mathrm{LN}$ size was $5.5 \mathrm{~mm}$. The sensitivity and specificity according to this optimal cutoff value were $64 \%$ and $75 \%$, respectively. In the $\mathrm{LN}$-positive group, the diameter of LNs in 5 patients were $<5.5 \mathrm{~mm}(29.4 \%, 5 / 17)$, in 7 patients were $\geq 10 \mathrm{~mm}(41.2 \%, 7 / 17)$, and in 5 patients were $5.5-10 \mathrm{~mm}(29.4 \%, 5 / 17)$.

\section{Discussion}

This study collected a cohort of ESCC patients who had undergone esophagectomy with left RLN LN dissection. The results suggest that the left RLN area is the high-risk area for LN metastasis. The LN size at CT is independently associated with left RLN LN metastasis. The RLN LNs $>5.5 \mathrm{~mm}$ at CT examination are more likely to be positive and should probably be dissected.

In this study, 2,505 LNs were removed from 94 ESCC participants during esophagectomy, which is similar to the mean of 24 RLN LNs per patient in a singlecenter study by van der Horst et al. (14) who shared a comparable level of surgery skill with us. This suggested that the lymphadenectomy method used at our hospital is comparable to that of other major institutions. Consistent with previous reports $(17,23,24)$, the LNPR was $5.2 \%$, and the LNMR was $45.7 \%$, indicating fairly accurate LN dissection. Furthermore, the main sites of the LN metastases were the left and right RLN (18.1\% and $14.9 \%$, respectively), peri-cardiac tissues (11.7\%), and left gastric artery $(13.8 \%)$, with the highest LNMR in the left RLN. Therefore, dissection of the left RLN LNs is essential. Unfortunately, 288 patients had to be excluded because they did not undergo left RLN LN dissection, suggesting that many surgeons are conservative in the removal of LNs in this area.

In this study, the esophagus-mobilized retraction method was used to harvest $380 \mathrm{LNs}$ of the left RLN area from 94 participants (mean of 4 left RLN LNs per participant), of which 30 were positive (LNPR 7.9\%). 
Table 1 Participant characteristics

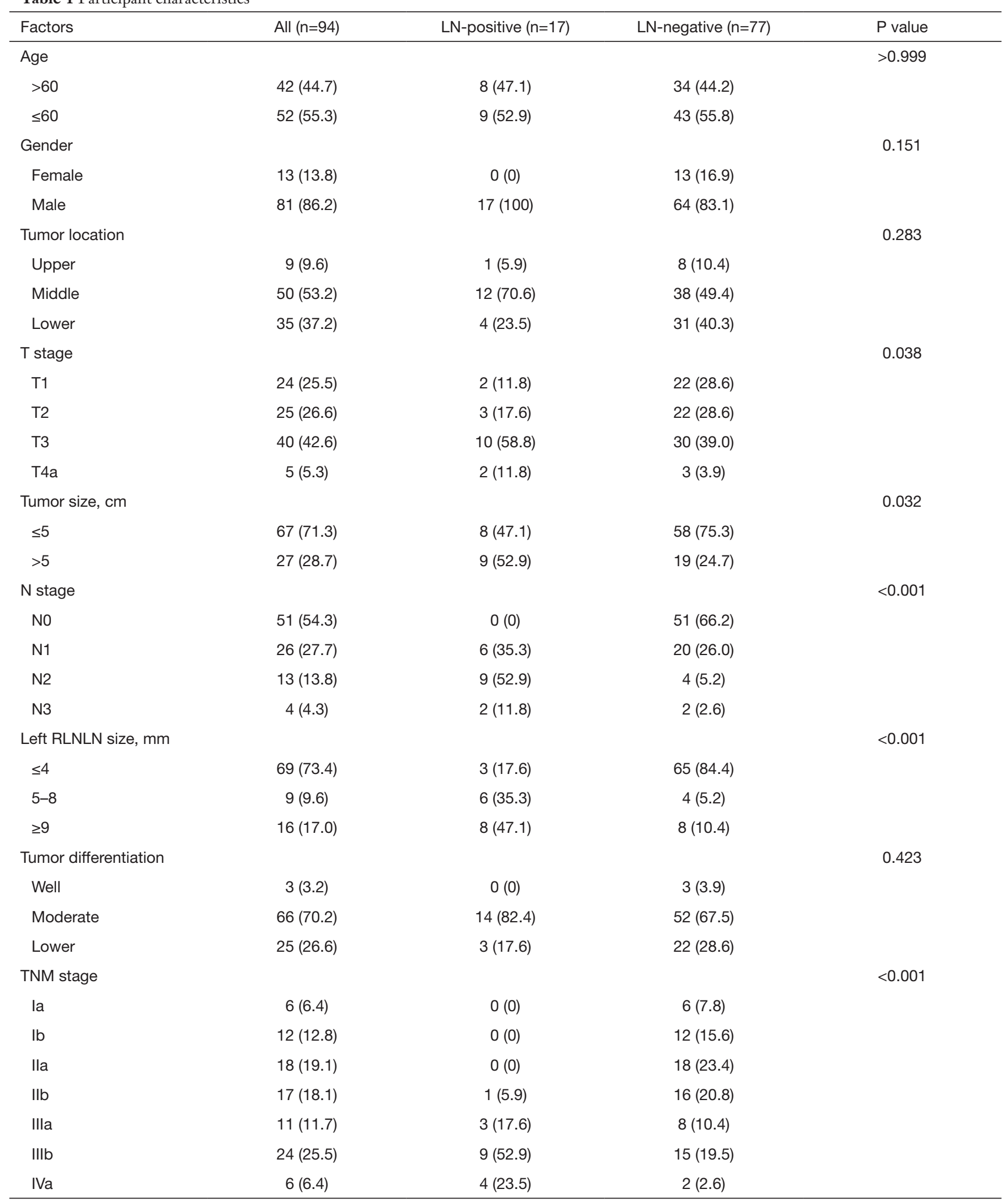

All data are presented as $\mathrm{n}(\%)$. LN, lymph node; RLN, recurrent laryngeal nerve; TNM, tumor node metastasis. 
Table 2 Logistic regression analysis of the factors associated with left RLN LN metastasis

\begin{tabular}{lcccccc}
\hline Factor & Regression coefficient & Standard error & Wald value & P value & OR & 95\% Cl \\
\hline T stage & 1.1445 & 0.9035 & 1.6 & 0.2053 & 4.161 & $0.259-6.001$ \\
Tumor size & 0.2049 & 0.1905 & 1.2 & 0.2820 & 0.818 & $0.128-1.614$ \\
N stage & 0.2261 & 0.9349 & 0.058 & 0.8089 & 1.798 & $0.639-3.142$ \\
LN size & 0.5606 & 0.1731 & 10.5 & 0.0012 & 1.569 & $0.259-1.956$ \\
TNM stage & 2.3383 & 1.3863 & 2.8 & 0.0917 & 1.111 & $0.325-3.412$ \\
\hline
\end{tabular}

RLN, recurrent laryngeal nerve; LN, lymph node; TNM, tumor node metastasis; OR, odds ratio; Cl, confidence interval.

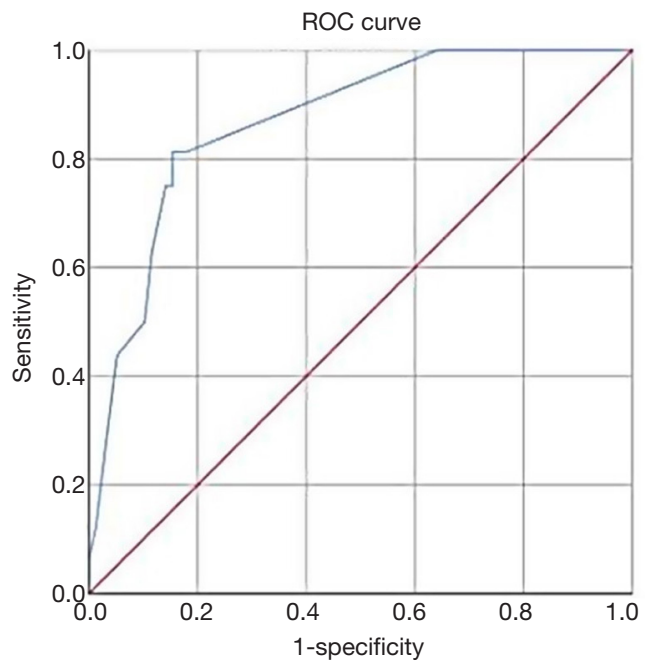

Figure 2 ROC analyses of the size of the largest left RLN (LN) at $\mathrm{CT}>5.5 \mathrm{~mm}$ for the prediction of metastasis in patients with ESCC. ROC, receiver operating characteristic; RLN, recurrent laryngeal nerve; LN, lymph node; CT, computed tomography; ESCC, esophageal squamous cell carcinoma.

Regarding the mean number of left RLN LNs, the results of the present study are consistent with Akagawa et al. (11). Regarding the LNMR of left RLN, the esophagus-mobilized retraction method (18.1\%) seems higher than that of the mesoesophageal suspension method (12.9\%) (25). The esophagus-mobilized retraction method might achieve a more in-depth dissection compared with the mesoesophageal suspension method, which could contribute to a higher LN yield. As far as complications are concerned, the complications in the present study were relatively few compared with those reported in the literature (14,25-27). Chylothorax and massive bleeding were not found in any of the 94 participants of this study. Therefore, the esophagus-mobilized retraction method to dissect left RLN LNs is probably worth popularizing.
As to the risk factors for left RLN LN metastasis, the univariable analyses showed that tumor size, $\mathrm{LN}$ size, $\mathrm{T}$ stage, $\mathrm{N}$ stage, and TNM stage were associated with positive RLN LNs, but that only the LN size was independently associated. Therefore, the size of the left RLN LNs probably plays an important role in predicting positive LNs. Using the ROC approach, the sensitivity and specificity of CT for diagnosing left RLN LN metastasis was optimal when the LNs were $\geq 5.5 \mathrm{~mm}$ in diameter. In the past, many surgeons believed that left RLN LNs $>10 \mathrm{~mm}$ had to be dissected (18), and the data about positive $\mathrm{LNs}<10 \mathrm{~mm}$ are therefore mostly missing. Nevertheless, using the $10 \mathrm{~mm}$ cutoff point most likely leaves many positive RLN LNs in place, which would mean that many patients would undergo palliative resection rather than radical resection. According to the cutoff value of $5.5 \mathrm{~mm}$, the diameter of the metastatic left RLN LNs in 5 patients were $<5.5 \mathrm{~mm}(29.4 \%, 5 / 17)$; that of 7 patients were $\geq 10 \mathrm{~mm}(41.2 \%, 7 / 17)$, and that of 5 patients were $5.5-10 \mathrm{~mm}(29.4 \%, 5 / 17)$. Because more than $70 \%$ of the metastatic left RLN LNs were larger than $5.5 \mathrm{~mm}$, it not nearly enough to only dissect left RLN LNs $>10 \mathrm{~mm}$. Nevertheless, positive LNs $<5.5 \mathrm{~mm}$ are still observed, but considering the poor prognosis and short survival of ESCC, it is currently unknown whether leaving those small positive RLN LNs will affect patient survival, but it is possible that it will decrease the risk of complications. This requires examination in future studies.

Furthermore, no metastases were observed in the subcarinal, supradiaphragmatic, common hepatic artery, and celiac artery LNs in these 94 participants. Tang et al. also reported that subcarinal LN dissection was not beneficial and could be omitted in superficial ESCC (28). Therefore, surgical removal of LNs in these areas may be over-treatment. Studies are still necessary to demonstrate the benefits and harms of dissection of LNs in different compartments. 
This study had some limitations. The number of participants was small, and they were from a single hospital. Because it was a retrospective study, a 1-to-1 comparison of each dissected LN to its initial CT image could not be performed. The results only suggest for now the possible predictive ability of the largest $\mathrm{LN}$ at CT to indicate the likelihood of finding at least one positive RLN LN. Finally, many patients were excluded because no RLN LNs were dissected. The results of this study will have to be confirmed through future multi-centered larger studies.

\section{Conclusions}

In conclusion, the size of the largest $\mathrm{LN}$ in left RLN area is an independent risk factor for metastasis in patients with ESCC. The results suggest that left RNL LNs $>5.5 \mathrm{~mm}$ on preoperative CT should be dissected.

\section{Acknowledgments}

Funding: This study was funded by the National Key Clinical Specialist Construction Programs of China (2013-544).

\section{Footnote}

Reporting Checklist: The authors have completed the STARD reporting checklist. Available at http://dx.doi.org/10.21037/ atm-21-377

Data Sharing Statement: Available at http://dx.doi. org/10.21037/atm-21-377

Conflicts of Interest: All authors have completed the ICMJE uniform disclosure form (available at http://dx.doi. org/10.21037/atm-21-377). The authors have no conflicts of interest to declare.

Ethical Statement: The authors are accountable for all aspects of the work in ensuring that questions related to the accuracy or integrity of any part of the work are appropriately investigated and resolved. All procedures performed in this study involving human participants were in accordance with the Declaration of Helsinki (as revised in 2013). The study was approved by the Ethics Committee of Tianjin Medical University Cancer Institute and Hospital (No. Bc2020176). The requirement for informed content was waived due to the retrospective nature of the study.
Open Access Statement: This is an Open Access article distributed in accordance with the Creative Commons Attribution-NonCommercial-NoDerivs 4.0 International License (CC BY-NC-ND 4.0), which permits the noncommercial replication and distribution of the article with the strict proviso that no changes or edits are made and the original work is properly cited (including links to both the formal publication through the relevant DOI and the license). See: https://creativecommons.org/licenses/by-nc-nd/4.0/.

\section{References}

1. NCCN Clinical Prcatice Guidelines in Oncology (NCCN Guidelines). Esophageal and Esophagogastric Junction Cancers. Version 4.2020. Fort Washington: National Comprehensive Cancer Network; 2020.

2. Allum WH, Blazeby JM, Griffin SM, et al. Guidelines for the management of oesophageal and gastric cancer. Gut 2011;60:1449-72.

3. Rustgi AK, El-Serag HB. Esophageal carcinoma. N Engl J Med 2014;371:2499-509.

4. Stahl M, Mariette C, Haustermans K, et al. Oesophageal cancer: ESMO Clinical Practice Guidelines for diagnosis, treatment and follow-up. Ann Oncol 2013;24 Suppl 6:vi51-6.

5. Bray F, Ferlay J, Soerjomataram I, et al. Global cancer statistics 2018: GLOBOCAN estimates of incidence and mortality worldwide for 36 cancers in 185 countries. CA Cancer J Clin 2018;68:394-424.

6. Ji X, Cai J, Chen Y, et al. Lymphatic spreading and lymphadenectomy for esophageal carcinoma. World J Gastrointest Surg 2016;8:90-4.

7. Akutsu $\mathrm{Y}$, Matsubara $\mathrm{H}$. The significance of lymph node status as a prognostic factor for esophageal cancer. Surg Today 2011;41:1190-5.

8. Smit JK, Pultrum BB, van Dullemen HM, et al. Prognostic factors and patterns of recurrence in esophageal cancer assert arguments for extended two-field transthoracic esophagectomy. Am J Surg 2010;200:446-53.

9. Shen Y, Zhang Y, Tan L, et al. Extensive mediastinal lymphadenectomy during minimally invasive esophagectomy: optimal results from a single center. J Gastrointest Surg 2012;16:715-21.

10. Tong D, Law S. Extended lymphadenectomy in esophageal cancer is crucial. World J Surg 2013;37:1751-6.

11. Akagawa S, Hosogi H, Yoshimura F, et al. Mesenteric excision for esophageal cancer surgery: based on the 
concept of mesotracheoesophagus. Int Cancer Conf J 2018;7:117-20.

12. Chiu CH, Wen YW, Chao YK. Lymph node dissection along the recurrent laryngeal nerves in patients with oesophageal cancer who had undergone chemoradiotherapy: is it safe? Eur J Cardiothorac Surg 2018;54:657-63.

13. Cuesta MA. Review of different approaches of the left recurrent laryngeal nerve area for lymphadenectomy during minimally invasive esophagectomy. J Thorac Dis 2019;11:S766-70.

14. van der Horst S, de Maat MFG, van der Sluis PC, et al. Extended thoracic lymph node dissection in roboticassisted minimal invasive esophagectomy (RAMIE) for patients with superior mediastinal lymph node metastasis. Ann Cardiothorac Surg 2019;8:218-25.

15. Sugawara K, Yamashita H, Uemura Y, et al. Preoperative lymph node status on computed tomography influences the survival of pT1b, T2 and T3 esophageal squamous cell carcinoma. Surg Today 2019;49:378-86.

16. Shen C, Liu Z, Wang Z, et al. Building CT Radiomics Based Nomogram for Preoperative Esophageal Cancer Patients Lymph Node Metastasis Prediction. Transl Oncol 2018;11:815-24.

17. Shi $Y, X u J$, Wang $Y$, et al. Prognostic significance of preoperative lymph node assessment for patients with stage pN0 esophageal squamous cell carcinoma after esophagectomy. J Thorac Dis 2019;11:732-43.

18. Dai Y, Tianwen X. Analysis of diagnosis of lymph node metastasis of esophageal carcinoma by CT compared with pathology. Cancer Res Clin 2014;26:169-71.

19. D'Amico TA. Mckeown esophagogastrectomy. J Thorac Dis 2014;6 Suppl3:S322-4.

20. Kim DJ, Park SY, Lee S, et al. Feasibility of a robotassisted thoracoscopic lymphadenectomy along the

Cite this article as: Chen $\mathrm{C}, \mathrm{Ma} Z$, Shang $\mathrm{X}$, Duan $\mathrm{X}$, Yue J, Jiang H. Risk factors for lymph node metastasis of the left recurrent laryngeal nerve in patients with esophageal squamous cell carcinoma. Ann Transl Med 2021;9(6):476. doi: 10.21037/ atm-21-377 recurrent laryngeal nerves in radical esophagectomy for esophageal squamous carcinoma. Surg Endosc 2014;28:1866-73.

21. Rice TW, Patil DT, Blackstone EH. 8th edition AJCC/UICC staging of cancers of the esophagus and esophagogastric junction: application to clinical practice. Ann Cardiothorac Surg 2017;6:119-30.

22. Rice TW, Kelsen DP, Blackstone EH. Esophagus and esophagogastric junction. In: Amin MB, editor. AJCC Cancer Staging Manuak, Eighth Edition. Chicago: American Joint Committee on Cancer; 2017.

23. Ye B, Zhong CX, Yang Y, et al. Lymph node dissection in esophageal carcinoma: Minimally invasive esophagectomy vs open surgery. World J Gastroenterol 2016;22:4750-6.

24. Yao F, Wang J, Yao J, et al. Is thoracoscopic-laparoscopic esophagectomy a better alternative to thoracoscopic esophagectomy? Int J Surg 2017;48:105-9.

25. Lin J, Kang M, Chen S, et al. Feasibility and strategy for left tracheobronchial lymph node dissection in thoracolaparoscopic esophageal cancer surgery. Thorac Cancer 2016;7:199-206.

26. Han ZY, Kang MQ, Lin JB, et al. Feasibility and strategy of common hepatic artery lymph node dissection in thoracolaparoscopic esophagectomy for thoracic esophageal squamous cell cancer. Medicine (Baltimore) 2018;97:e13587.

27. Chao YK, Hsieh MJ, Liu YH, et al. Lymph Node Evaluation in Robot-Assisted Versus Video-Assisted Thoracoscopic Esophagectomy for Esophageal Squamous Cell Carcinoma: A Propensity-Matched Analysis. World J Surg 2018;42:590-8.

28. Tang H, Tan L, Wang H, et al. Is Routine Subcarinal Lymph Node Dissection Necessary in Superficial Esophageal Squamous Cell Carcinoma? A Propensity Score Matching Analysis. J Cancer 2019;10:2350-6. 

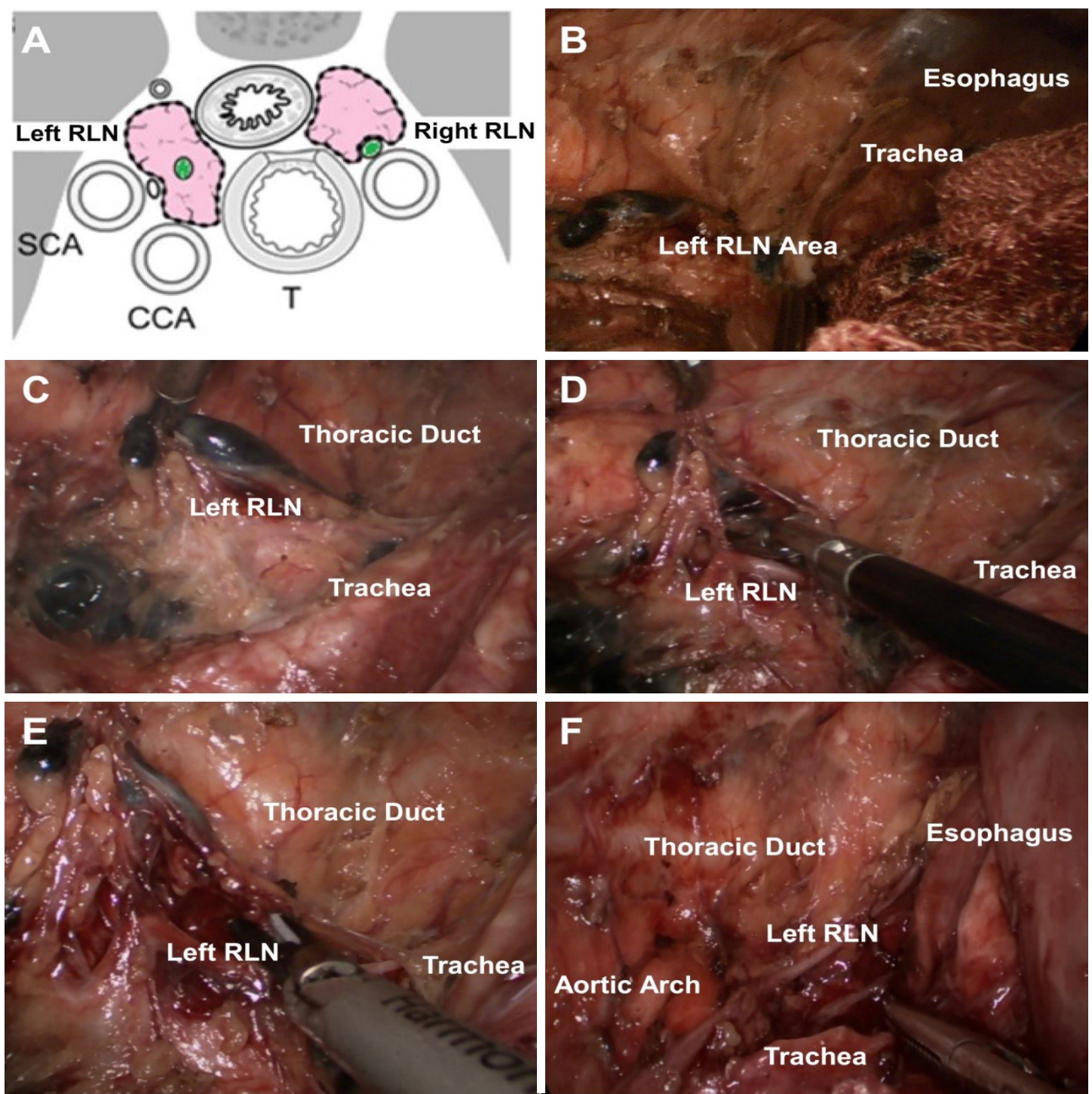

Figure S1 The esophagus-mobilized retraction procedure. (A) The extent of lymphadenectomy along the left RLN (red area). The green areas are the left and right RLN. (B) Retracting the esophagus mobilized with a loop and rotating the trachea to the right with a 5-lobed liver retractor to expose the left RLN area. (C) The pretracheal soft tissue was dissociated along the anterior trachea by an electric hook and lifted to expose the left RLN. (D) Forceps were used to hollow the soft tissue of the left RLN. (E) An ultrasonic scalpel or scissors or Maryland bipolar forceps was used to cut the soft tissue and LNs of left RLN. (F) The left RLN in the skeletal state. RLN, recurrent laryngeal nerve; CCA, left common carotid artery; SCA, left subclavian artery; T, trachea. 
Table S1 Status of the dissected regional LNs

\begin{tabular}{|c|c|c|c|}
\hline Field & Location & LNMR (\%) & LNPR (\%) \\
\hline \multirow{6}{*}{ Thoracic LN } & Left recurrent nerve LN & $18.09(17 / 94)$ & $7.89(30 / 380)$ \\
\hline & Right recurrent nerve LN & $14.89(14 / 94)$ & $7.99(23 / 288)$ \\
\hline & $\begin{array}{l}\text { LN between left low paratracheal and main } \\
\text { pulmonary artery }(4 \mathrm{~L})\end{array}$ & $2.13(2 / 94)$ & $4.29(3 / 70)$ \\
\hline & Left main bronchus LN & $2.13(2 / 94)$ & $6.78(4 / 59)$ \\
\hline & Right main bronchus LN & $1.06(1 / 94)$ & $4.76(1 / 21)$ \\
\hline & Middle paraesophageal LN & $4.26(4 / 94)$ & $5.77(6 / 104)$ \\
\hline \multirow{5}{*}{ Abdominal LN } & Left gastric artery LN & $13.83(13 / 94)$ & $7.23(17 / 235)$ \\
\hline & Lesser curvature LN & $3.19(3 / 94)$ & $2.68(4 / 149)$ \\
\hline & Common hepatic artery LN & $0(0 / 94)$ & $0(0 / 40)$ \\
\hline & Splenic artery LN & $1.06(1 / 94)$ & $2.08(1 / 48)$ \\
\hline & Celiac artery LN & $0(0 / 94)$ & $0(0 / 38)$ \\
\hline
\end{tabular}

In some regions, the number of LNs is less than the number of patients, indicating that these regions were cleaned, but no LNs were found during surgery. LN, lymph node; LNMR, lymph node metastasis rate; LNPR, lymph node positive rate. 